\title{
Severe skin complications in patients treated with antidepressants: a literature review
} \author{
Maria Gałuszko-Węgielnik ${ }^{1}$, Jerzy Landowski ${ }^{1}$ \\ ${ }^{1}$ Department of Psychiatry, Medical University of Gdansk, Poland \\ Head of Department: Prof. Jerzy Landowski MD, PhD \\ ${ }^{2}$ Department of Dermatology and Allergology, Medical University of Gdansk, Poland \\ Head of Department: Prof. Roman Nowicki MD, PhD
}

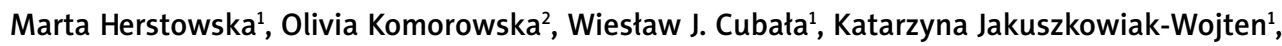

Postep Derm Alergol 2014; XXXI, 2: 92-97

DOI: $10.5114 /$ pdia.2014.40930

\begin{abstract}
Clinical manifestations of drug-induced skin reactions include a wide range of symptoms, from mild drug-induced exanthemas to dangerous and life-threatening generalized systematic reactions. Adverse drug reactions of low risk include phenomena such as drug-induced rashes, phototoxic reactions, eczemas and urticarias, which appear most often when the medication is being introduced. Drug-induced skin reactions to psychotropic medication are usually associated with antiepileptic drugs. However, a significant role can be assigned to selective serotonin reuptake inhibitors. The aim of this paper is to review a spectrum of severe skin complications in patients treated with antidepressants with the indication of their clinical monitoring and management.
\end{abstract}

Key words: adverse drug reactions, drug hypersensitive syndrome, antidepressants, selective serotonin reuptake inhibitors.

\section{Introduction}

Clinical manifestations of drug-induced skin reactions include a wide range of symptoms, from mild drug-induced exanthemas to dangerous and life-threatening generalized systematic reactions. Adverse drug reactions of low risk include phenomena such as drug-induced rashes, phototoxic reactions and eczemas, which appear most often when the medication is being introduced. In most cases, the reactions go away on their own, thus do not need the medication to be discontinued and there is no need for more serious medical interventions. The aim of this paper is to review a spectrum of severe skin complications in patients treated with antidepressants.

In the case of using the antidepressant drugs on such a huge scale it is worth paying attention to and highlighting possible unwanted effects, which despite a good reaction to the medicine, may be contraindications to such pharmacotherapy. At first, unwanted, severe skin symptoms connected with antidepressant treatment concerned only tricyclic drugs. Together with the development of pharmacotherapy and introduction of innovative preparations, new information concerning the drugs from SSRI groups arose. Regardless of few publications, it is worth presenting descriptions of cases of severe skin complications after antidepressants as those situations seem more frequent and their diagnosis still causes problems.

\section{Severe skin complications associated with antidepressants use}

Clinicians become increasingly interested in the highrisk skin reactions including:

1. Erythema multiforme (EM);

2. Stevens-Johnson syndrome (SIS);

3. Lyell's syndrome (toxic epidermal necrolysis - TEN);

4. Acute generalized exanthematous pustulosis (AGEP);

5. Drug-induced hypersensitivity syndrome (DIHS).

The first three disease syndromes are considered to be three severities of the same pathological syndrome [1].

\section{Erythema multiforme}

Because of a variety of clinical presentation, erythema exudativum multiforme causes a lot of diagnostic problems. We can distinguish two forms - minor, severe (Stevens-Johnson syndrome) and toxic (Lyell's syndrome).

Address for correspondence: Marta Herstowska MD, PhD, Department of Dermatology and Allergology, Medical University of Gdansk, 7 Dębinki St, 80-952 Gdansk, Poland, phone: +48 608327 141, e-mail: eziat1@wp.pl Received: 19.03.2013, accepted: 17.09.2013. 
The clinical presentation of the minor form is characterized by EM placed both on skin and on mucosa of the whole body. The skin changes are characterized by erythema and edema and are often well separated from the healthy skin. Illness efflorescence is often multicolour, mostly red and grey, forming circinate, concentric figures (resembling shooting range) (Figure 1). Skin changes can be mainly located on distal parts of upper and lower limbs, they may also appear on mouth mucosa and in the area of genitourinary organs. Those changes may be accompanied by itching and burning [2-4].

Erythema multiforme is formed as a consequence of hypersensitivity of human body to different factors: viral (mainly Herpes simplex type I and II), bacteria (mainly streptococcus), chemical and medicine ones (salicylates, antibiotics, sulphonamides, barbiturates). It rarely appears in children below the age of three and in elderly people.

While treating EM connected with pharmacotherapy it is crucial to discontinue the drug that is being used. Additionally, histamine antagonists are being incorporated and regionally inert creams and glucocorticoid preparations of low strength are being used. For places with no epidermis we use disinfecting preparations, sometimes together with glucocorticoids [5].

In the literature, the first information about the development of EM appeared in the 1980s and applied to mianserin. A few years later, a case of erythema appearance after trazodone [6] and bupropion was described. In recent years, its development has been noticed after drugs from the SSRI sertraline group [7].

\section{Stevens-Johnson syndrome and Lyell's syndrome}

Stevens-Johnson syndrome and TEN are life-threatening multiorgan syndromes. They are characterized by dying of skin epidermis, mucosa laceration and inner organ reaction. Their pathophysiology is connected to keratinocyte apoptosis caused by disorder on the level of the FAS receptor and its ligand [8-10]. Another theory regarding the pathogenesis of SJS/TEN points out to perforin being released from lymphocytes, which in low concentration, activates apoptosis and in high concentration, causes skin epidermis necrosis.

Until today some 100 treatments have been identified as those causing SJS and TEN. According to Rawlins and Thomas' distinction, two types of negative response to treatment may be described. Type I (toxic reactions) is connected with the applied dosage, its unwanted symptoms or matching with other chemical substances - these are the reactions that can be foreseen. Type II (idiosyncratic reactions) appears in hypersensitive patients - these are unforeseeable symptoms. They may be connected to an allergy to the medicine, with enzymatic deficiency, or intolerance to the drug. Type $\|$ reactions are more rare and include SJS and TEN [11].

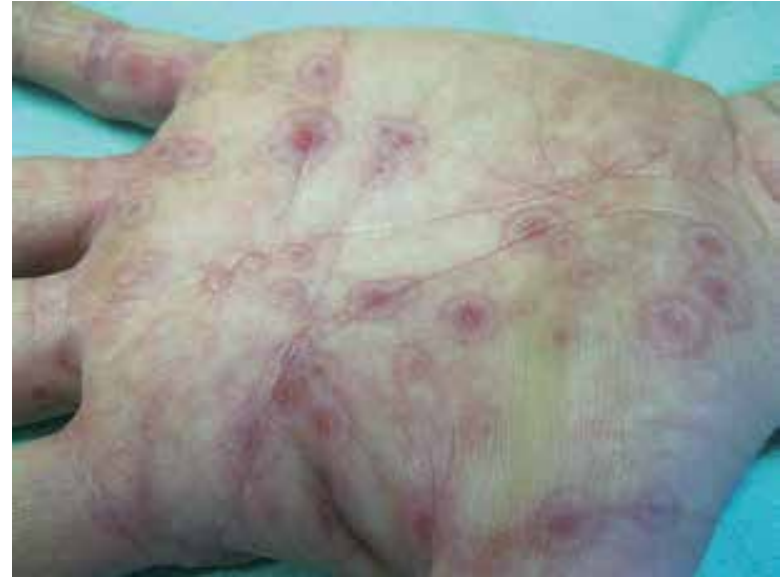

Figure 1. Erythema multiforme

The frequency of SJS cases is about 1.6 million with a mortality rate of $5 \%$, and of TEN is $0.4-1.2$ million per year, with a mortality rate of $35 \%$. The distinction of blister lesions is based on the degree of skin damage. In SJS it involves < $10 \%$ of the body area, and is accompanied by increased ulceration in the area of oral cavity's and crotch's mucosa. In Lyell's syndrome, the blister lesions cover $>30 \%$ of the body. Ten - thirty percent can be identified as the superimposed syndrome of SJS/TEN [12, 13].

Those syndromes begin in a non-specific way. Patients may experience flu-like symptoms. The first changes appear in the surroundings of mucosa. On the skin a measles-like papulopustular eruption may appear. Occasionally, the prodromal period may appear as pneumonia, nephritis or myocarditis. Within 2 to 14 days the rash develops into erythema multiforme. Skin becomes heated and cracks. While the disease develops, big and slack blisters full of liquid start to appear, which afterwards lead to ulceration and erosion. This process may spread onto the mucosa of the digestive system, respiratory system, urinary system and conjunctiva (Figure 2).

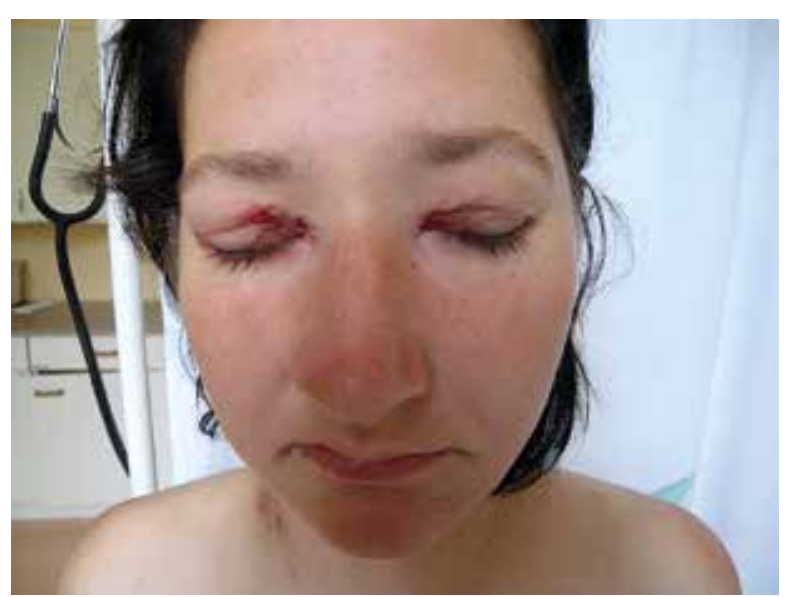

Figure 2. Stevens-Johnson syndrome 


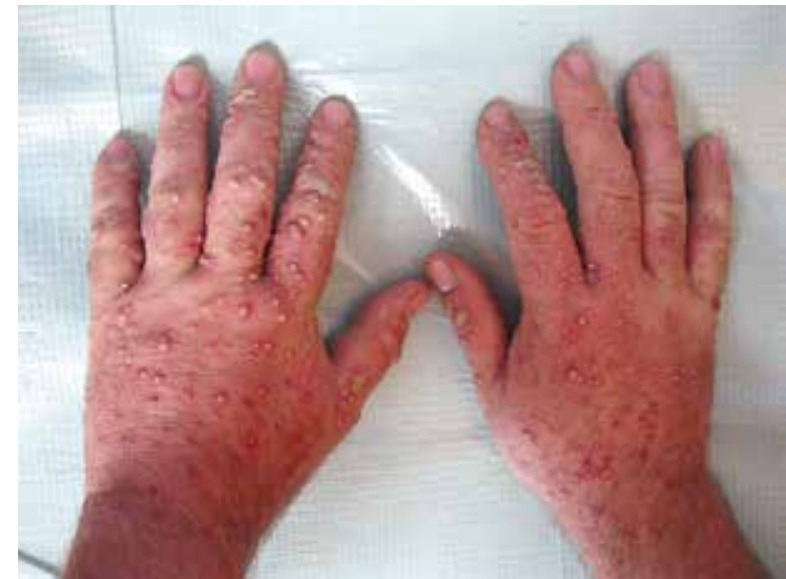

Figure 3. Acute generalized exanthematous pustulosis

Skin lesions are accompanied by pain, fever and chills. Complications may be of multiorgan nature and concern kidneys, liver, pancreas and bone marrow [14, 15]. In TEN, more severe organ symptoms occur. A dangerous respiratory failure may develop in the area of the respiratory system as diverticulosis in the digestive system.

Treatment of those life-threatening severe drug reactions should be carried out in specialized facilities. It is most important to even the disorders of homeostasis and prevent any infections. Within 1-4 days intravenous immunoglobulin, which inhibits keratinocyte apoptosis, has to be administered (0.8-1.2 $\mathrm{mg} / \mathrm{kg}$ of body mass). When the skin is affected in $<25 \%$ treatment combined with steroids can be used.

One of the first information about the possible occurrence of SJS/TEN after antidepressant drugs appeared in the 1990s and took into account all medications from the SSRI group: fluoxetine [16] and sertraline [17]. In 1994, Wolkenstein, Cremniter and Roujeau reported the first patient who developed severe TEN after starting paroxetine (30 mg/day). A 23-year-old female was hospitalized for depression with psychotic features. After 2 weeks she developed a widespread febrile bullous eruption with mucous membrane involvement. Histological examination of the skin showed total necrosis of the epidermis consistent with the diagnosis of TEN [18]. In 2008, two publications appeared, which concerned the development of a full-blown TEN syndrome after drugs from the SSRI group - fluoxetine and paroxetine. The first case report described a 34-year-old patient associated with the addition of fluoxetine for a major depressive episode with psychotic features [19]. The second one presented an 80-year-old female started on paroxetine of $10 \mathrm{mg}$ per day, who had extensive vesiculobullous skin eruptions. As a result she started i.v. fluid and corticosteroid therapy with a positive effect. The TEN diagnosis was based on skin biopsy [20]. Two years later, SJS appearance was described in a 56-year-old patient treated with bupropi- on because of an episode of depression diagnosed [21]. A great interest has risen after a publication concerning induction of SJS as a consequence of treatment with mirtazapine [22]. Previous years revealed new reports concerning possibilities of SJS symptoms appearing after mirtazapine [23].

\section{Acute generalized exanthematous pustulosis}

Acute generalized exanthematous pustulosis (drug-induced pustular rash and pustulosa) is a rare, severe and adverse drug reaction. Its prevalence rate is estimated from 0.35 to 5 cases per million per year [24].

The AGEP is characterized by rapid appearance of pustular skin lesions on the whole body. Pustules are filled with yellow fluid and they are not associated with hair follicles (Figure 3). In the course of the pustular drug eruption the pustules are ruptured with forming of erosions and crusts with exfoliation of the skin above. Skin lesions are not on the palms and soles and they are very rare on the mucous membranes. Skin lesions are associated with fever, and laboratory tests reveal neutropenia. Acute generalized exanthematous pustulosis may last for 1 to 2 weeks. The histopathological picture shows intraepidermal or subcorneal pustules filled with neutrophils, perivascular inflammatory infiltration and edema of the papillary dermis.

In the course of the AGEP, it is the most important to identify the suspected drug. The disease may resolve spontaneously within 3 weeks after instant recognition of the suspicious drug. The most popular treatment of the AGEP is topical therapy involving inhaled and fluid corticosteroids. Protection against secondary bacterial infection plays an important role in this skin condition.

There have been a few reports of the AGEP in psychiatric patients treated by antidepressants so far. Two cases of this severe skin condition were noticed after administration of amoxapine - tetracyclic antidepressant $[25,26]$.

Pustular drug eruption can be induced by selective serotonin reuptake inhibitors - a group of new antidepressants. A case of 77-year-old man with AGEP 6 days after sertraline administration is observed. It was a very severe reaction with mucous membrane involvement and inflammation. Interestingly, the skin lesions were significantly intensified on sun-exposed areas [27].

\section{Drug-induced hypersensitivity syndrome}

The diagnosis of DIHS gives rise to many doubts and clinical difficulties. The above-mentioned syndrome is rarely diagnosed and is among misdiagnosed idiopathic skin disorders together with erythroderma. The drug-induced reaction with systematic symptoms is characterized by maculopapular skin changes, which are accompanied by three symptoms: lymphadenopathy, 
hematological disorders and infiltration of inner organs. In contemporary literature we can come across a few names of reactions of this type: drug-induced erythema with eosinophilia and systematic symptoms (DRESS) and drug-induced delayed multiorgan hypersensitivity syndrome (DIDMOHS) [28].

The diagnostic criteria of DRESS by Shiohan (for the syndrome to be recognized, 5 out of 6 criteria need to be fulfilled) include:

1. Maculopapular exanthema appearing after 3 weeks of the chosen drug therapy;

2. Lymphadenopathy;

3. Fever;

4. Leukocytosis (> $10 \times 10$ to the power of $9 / 1)$ :

- Atypical lymphocytosis,

- Eosinophilia;

5. Hepatitis;

6. Reactivation of HHV-6.

For the first time DRESS was described as a reaction to antiepileptic drugs. Among other groups of drugs, a significant role can be assigned to antidepressant drugs (both tricyclic antidepressants and selective serotonin reuptake inhibitors), sulfonamides, ant-inflammatory drugs, antiviral drugs, ACE-inhibitors and $\beta$-blockers. The incidence of this syndrome ranges from $1: 1000$ to $1: 10000$ per drug exposure - which is more often than SIS.

The most common symptom at the beginning of DRESS syndrome is fever $\left(38-40^{\circ} \mathrm{C}\right)$ occurring in 90$100 \%$ of cases, and rash ( $87 \%$ of cases). At first, the rash appears on the face, torso, upper limbs and after some time it moves onto the lower parts of the body. Disease efflorescence looks like maculopapular erythema with severe skin itching, which over time may transform into erythroderma and in some cases lead to changes typical of SIS and TEN (Figure 4). The face rash is often accompanied by face swelling, inflammation of conjunctiva and laceration in the area of mucosa. Skin changes do not exactly show the degree of inner organ infection. Most often changes appear in the liver, which may only be an asymptomatic rise of enzymatic parameters, in more severe cases it may lead to hepatosplenomegaly, granulomatous liver infection or necrotic hepatitis fulminans [29]. More seldom one may encounter changes in the kidneys, respiratory system and central nervous system. Occasionally, the lymphocytic infiltrate contains atypical cells or is dense enough to raise the diagnosis of cutaneous lymphoma [30]. Mortality with the DHS reaches $10 \%$ and as one of the most common causes of death we should mention changes in liver, coagulation disorder and sepsis.

In DRESS syndrome treatment, the main role can be assigned to systematic glucocorticoids in dosage of $0.5-1.0 \mathrm{mg} / \mathrm{kg}$ of body mass. It is important to observe the patient to see if any inner organ changes appear. In case of the kidneys we administer prednisone in a dose of $1-2 \mathrm{mg} / \mathrm{kg}$ of body mass for 1 to 3 months, and in case

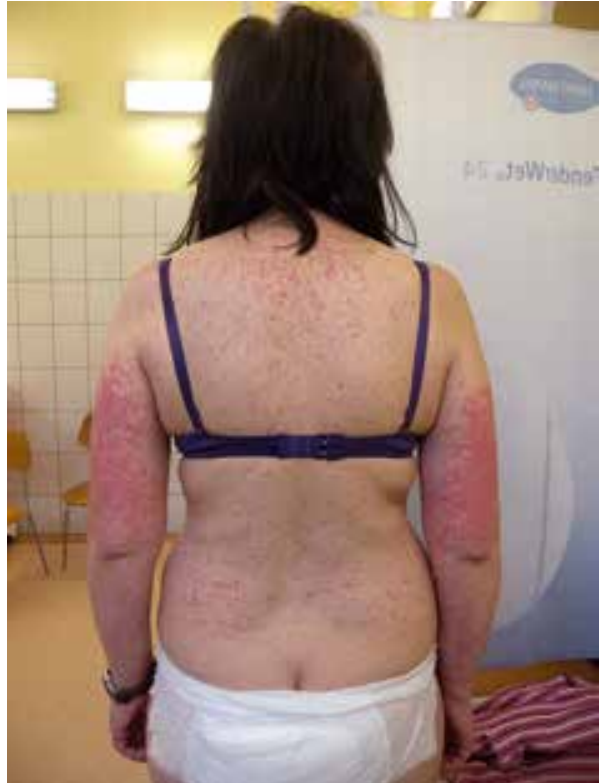

Figure 4. Drug reaction with eosinophilia and systemic symptoms

of lung changes we use methylprednisolone pulses of $1 \mathrm{~g}$ per day for 3 days. Skin changes often require local use of glucocorticoids.

The pathomechanism of DRESS has not yet been fully identified, but it is said that the drug-induced symptoms are connected with the dysfunction of the $\mathrm{P}_{450}$ cytochrome, and biologically reactive metabolites of drugs $[31,32]$ that are present in the bloodstream. Those metabolites can affect the activation of macrophages, eosinophilia, and T lymphocytes. This is combined with releasing cytokines, mainly the IL-5, from those cells [33]. Another reason is the possibility of reactivation of certain viruses in the outcome of DIHS, mainly HHV-6 and HHV-7, EBV, CMV. This is combined with the process of replication of the viral DNA through awoken immunocompetent cells [34]. In patients in whom DRESS symptoms have been observed, a rise of IgG serum concentration against the virus can be seen after 3 weeks of symptoms' occurrence. Moreover, the virus's DNA can be discovered in patients' blood and skin using the PCR method. The replication of the virus needs prior activation of T lymphocytes, which happens most likely with the use of drugs or their reactivated metabolites. It is also worth mentioning that the reaction of HHV-6 may cause a longer and more severe outcome of the hypersensitivity syndrome for a certain drug.

Until recently the main disadvantage in recognizing DRESS syndrome was the lack of sensitive and specific laboratory tests. The newest results show that the diagnostic meaning is shown by an increase in IgG antibody titer against HHV-6 and disambiguation of HHV-6 DNA [35]. In cases of a more mild outcome, those results may be negative. Positive results are achieved in the $3^{\text {rd }}-4^{\text {th }}$ 
week from the beginning of symptoms. Research to confirm the reactivation of viral infections often bring about great diagnostic quality. The diagnostic markers include in high concentration 2',5-oligoadenylate synthesis connected with the immunological disorder as a result of HHV-6 reactivation [36]. In order to verify the etiology of DRESS syndrome, the use patch tests and lymphocyte transformation tests are the acknowledged diagnostic methods used with drug hypersensitivity.

In 2001, some publications described the appearance of hypersensitivity syndrome with systematic symptoms after citalopram and fluoxetine [37]. It seems that this publication has shown a new point of view on DRESS diagnosis. Because of initially unspecific image of clinical drug-induced hypersensitive disorders in DRESS diagnosis, we have to rule out drug-induced lupus erythematosus, mononucleosis, Kawasaki disease, SJS, toxic epidermal necrolysis, measles, pseudolymphoma, immunoblastic lymphadenopathy, serum sickness-like reaction and Staphylococcal toxic shock syndrome. The onset of symptoms of DRESS usually occurs 2-8 weeks after drug administration, which is longer than for other drug reactions. Severe cutaneous adverse reactions to drugs are groups of drug hypersensitivity reactions with a heterogeneous clinical presentation. Although the prevalence of these reactions is low, they may result in prolonged hos pitalization, substantial disability, and even death. Medical journals publish articles about severe complications of DRESS syndrome after amitriptyline [38]. Hypersensitivity syndrome after amitriptyline includes skin rash and fever, very often with hepatitis, arthralgia, lymphadenopathy and hematological disorders (mainly eosinophilia and atypical lymphocytosis). This publication presents a case of a 24-year-old woman who experienced hypersensitivity syndrome 3 weeks after the initiation of amitriptyline (50 mg daily). In the same year, a publication described the occurrence of DRESS syndrome with rare complications in the respiratory system after clomipramine [39].

\section{Conclusions}

Psychotropic agents such as antidepressants, mood stabilizing drugs and anxiolytics, have been in widespread use since mid-1950s. Among the most common side effects associated with their use are those involving the skin. It is estimated that skin reactions caused by psychotropic drugs are twice as frequent as those caused by other drugs.

In spite of progress in the therapy of depression, new problems emerge. Adverse cutaneous effects of selective serotonin reuptake inhibitors are rare but the knowledge of these reactions is very important. Different serotonin uptake blockers could be involved in the same allergic reaction, suggesting cross reactivity, although these drugs have different chemistry structures. After an adverse ef- fect occurs, it is advisable to substitute a medication by one of the other classes of antidepressants.

There is no doubt that drugs that act in a selective way are safer than tricyclic antidepressants. However, the risk of unwanted skin reactions does not seem to be dependent on selectivity against specific receptors. It depends more on the specific features of the patient. One of them is the age - it has been noticed that the majority of unwanted drug-induced reactions appear in young children and in people above the age of 65 years. Moreover, in elderly patients we may more often encounter coexistence of other somatic diseases and as a result, with acquiring many drugs. Over-the-counter drugs, like nonsteroidal anti-inflammatory drugs, can often hide the real factor of skin complications. Such drug-induced skin reactions can appear as well with other constitutional or infectious illnesses or environmental factors like smoking or abuse of alcohol.

In conclusion, it seems that individualization of pharmacotherapy is crucial, together with regular evaluation of safety and tolerance of the treatment. Moreover, the patient has to be aware of symptoms, which may be a sign of developing hypersensitivity to a specific drug as severe skin complications belong to the spectrum of its adverse drug reactions.

The paper emphasizes the significance of patients' education about the drug-associated severe skin complications and the importance of their early management along with the need of constant evaluation of treatment safety and tolerance. Physicians should be vigilant to severe skin complications that belong to the spectrum of adverse drug reactions to selective serotonin reuptake inhibitors.

\section{Conflict of interest}

All authors declare no conflict of interest.

\section{References}

1. Wilkins J, Morrison L, White CR Jr. Oculocutaneoous manifestations of erythema multiforme/Stevens-Johnson syndrome/toxic epidermal necrolysis spectrum. Dermatol Clin 1992; 10: 571.

2. Forman R, Koren G, Shear NH. Erythema multiforme, Stevens-Johnson syndrome and toxic epidermal necrosis in children. A review of 10 years' experience. Drug Safety 2002; 25: $965-72$.

3. Wolf R, Matz H, Marcos B, Orion E. drug rash with eosinophilia and systemic symptoms vs toxic epidermal necrolysis: the dilemma of classification. Clin Dermatol 2005; 23: 311-4.

4. Mockenhaupt M, Wessenheimer J, Tennis P, Schlingmann J. Risk of Stevens-Johnson syndrome and toxic epidermal necrolysis in new users of antiepileptics. Neurology 2005; 64: 1134-8.

5. Czarnecka-Operaca M, Silny P. Drug eruptions. Alergia Astma Immunologia 2000; 5: 165-74. 
6. Ford HE, Jenike MA. Erythema multiforme associated with trazodone therapy. J Clin Psychiatry 1985; 46: 294-5.

7. Khan MA, Fitzgerald K. Low-dose sertraline-induced erythema multiforme in a child. J Child Adolesc Psychopharmacol 2012; 22: 179-80.

8. Viard I, Wehrli P, Bullani R. Inhibition of toxic epidermal necrolysis by blockade of CD95 with human intravenous immunoglobulin. Science 1998; 282: 490-3.

9. Wehrli P, Viard I, Bullani R. Death receptors in cutaneous biology and disease. J Invest Dermatol 2000; 115: 141-8.

10. Abe R, Shimizu T, Shibaki A. Toxic epidermal necrosis and Stevens-Johnson syndrome are induced by soluble Fas ligand. Am J Pathol 2003; 162: 1515-20.

11. Rawlings M, Thompson W. Mechanism of adverse drug reac tions. Textbook of adverse drug reactions. Oxford University Press 1991; 18-45.

12. Merck HF. Clinical aspects of drug related allergies [Polish]. Alergia 2002; 12.

13. Auquiler-Dunant A, Mockenhaupt M, Naldi L. Correlations between clinical patterns and causes of erythema multiforme majus, Stevens-Johnson syndrome and toxic epidermal necrolysis: result of international prospective study. Arch Dermatol 2002; 138: 1019-24.

14. Fritsch PO, Sideroff A. Drug-induced Stevens-Johnson syndrome/toxic epidermal necrolysis. Am J Clin Dermatol 2000; 1: 349-60.

15. Prendiville J. Stevens-Johnson syndrome and toxic epidermal necrolysis. Adv Dermatol 2002; 18: 151-73.

16. Bodokh I, Lacour JP, Rosenthal E, et al. Lyell syndrome or toxic epidermal necrolysis and Stevens-Johnson syndrome after treatment with fluoxetine. Therapie 1992; 47: 441.

17. Jan V, Toledano C, Machet L, et al. Stevens-Johnson syndrome after sertraline. Acta Derm Venereol 1999; 79: 401.

18. Wolkenstein P, Cremniter D, Roujeau. Toxic epidermal necrolysis after paroxetine treatment. Eur Psychiatry 1995; 10: 162.

19. Jonsson GW, Moosa MY, Jeenah FY. Toxic epidermal necrolysis and fluoxetine: a case report. J Clin Psychopharmacol 2008; 28: 93-5.

20. Ahmed R, Eagleton C. Toxic epidermal necrolysis after paroxetine treatment. N Z Med J 2008; 121: 86-9.

21. Surovik J, Riddel C, Chon SY. A case of bupropion-induced Stevens-Johnson syndrome with acute psoriatic exacerbation. J Drugs Dermatol 2010; 9: 1010-2.

22. Belkahia A, Hillaire-Buys D, Dereure O, et al. Stevens-Johnson syndrome due to mirtazapine-first case. Allergy 2009; 64: 1554.

23. Bhasin A, Gurtoo A, Gupta L, Aggarwal G. First case of mirtazapine - induced Stevens - Johnson syndrome from India. Indian J Pharmacol 2012; 44: 656-8.

24. Sidoroff A, Dunant A, Viboud C, et al. Risk factors for acute generalized exanthematous Pustulosis (AGEP) - results of a multinational case-control study. Br J Dermatol 2007; 157: 989-96.

25. Loche F, Durieu C, Bazex J. Acute generalized exanthematous pustulosis induced by amoxapine. Acta Derm Venereol 1998; 78: 224.

26. Larbre B, Kanitakis J, Savy C, et al. Acute exanthematous pustulosis during amoxapine treatment. Ann Dermatol Venereol 1994; 121: 40-1.

27. Thédenat B, Loche F, Albes B, et al. Acute generalized exanthematous pustulosis with photodistribution pattern induced by sertraline. Dermatology 2001; 203: 87-8.
28. Sullivan J, Shear N. The drug hypersensitivity syndrome. Arch Dermatol 2001; 137: 357-64.

29. Knowles S, Shapiro L, Shear N. Anticonvulsant hypersensitivity syndrome. Drug Safety 1999; 21: 489-501.

30. Roujeau JC. Clinical heterogeneity of drug hypersensitivity. Toxicology 2005; 209: 123-9.

31. Saida S, Yoshida A, Tanaka R, et al. A case of drug-induced hypersensitivity syndrome-like symptoms following HHV-6 encephalopathy. Allergol Int 2010; 59: 83-6.

32. Higuchi M, Agatsuma T, lizima $M$, et al. A case of drug-induced hypersensitivity syndrome with multiple organ involvement treated with plasma exchange. Ther Apher Dial 2005; 9: 412-6.

33. Pichler WJ. Delayed drug hypersensitivity reactions. Ann Intern Med 2003; 139: 683-93.

34. Shiohara T, Kano Y. A complex interaction between drug allergy and viral infection. Clin Rev Allergy Immunol 2007; 33: 124-33.

35. Watanabe T, Nakashima $\mathrm{H}$, Ohmatsu $\mathrm{H}$, et al. Detection of human herpesvirus- 6 transcripts in carbamazepine-induced hypersensitivity syndrome by in situ hybridization. J Dermatol Sci 2009; 54: 134-6.

36. Kawakami T, Fujita A, Takeuchi S, et al. Drug-induced hypersensitivity syndrome: drug reaction with eosinophilia and systemic symptoms (DRESS) syndrome induced by aspirin treatment of Kawasaki disease. J Am Acad Dermatol 2009. 60: 146-9.

37. Richard MA, Fiszenson F, Jreissati M, et al. Cutaneous adverse effects during selective serotonin reuptake inhibitors therapy: 2 cases. Ann Dermatol Venereol 2001; 128: 759-61.

38. Milionis HJ, Skopelitou A, Elisaf MS. Hypersensitivity syndrome caused by amitriptyline administration. Postgrad Med J 2000; 76: 361-3.

39. Gallego J, Hauss PA, Salaün M, et al. Clomipramine hypersensitivity with predominantly pulmonary involvement. Rev Mal Respir 2012; 29: 430-4. 